\title{
Comparison of Human Interpretation and a Rule-Based Algorithm for Instrumented Sit-to-Stand Test
}

\author{
Hee-Won Jung ${ }^{1, *}$, Seongjun Yoon ${ }^{2, *}$, Ji Yeon Baek ${ }^{1}$, Eunju Lee ${ }^{1}$, Il-Young Jang ${ }^{1}$, Hyunchul Roh ${ }^{2}$ \\ ${ }^{1}$ Division of Geriatrics, Department of Internal Medicine, Asan Medical Center, University of Ulsan College of Medicine, Seoul, Korea \\ ${ }^{2}$ Dyphi Research Institute, Dyphi Inc., Daejeon, Korea
}

\author{
Corresponding Author: \\ Il-Young Jang, MD \\ Division of Geriatrics, Department of \\ Internal Medicine, Asan Medical \\ Center, University of Ulsan College of \\ Medicine, 88 Olympic-ro 43-gil, \\ Songpa-gu, Seoul 05505, Korea \\ E-mail: onezero2@gmail.com \\ ORCID:
}

https://orcid.org/0000-0003-3617-3301

Hyunchul Roh, $\mathrm{PhD}$

Dyphi Research Institute, Dyphi Inc.,

233, Dae-hak-ro, Yuseong-gu, Daejeon

34139, Korea

E-mail: roh@dyphi.com

ORCID:

https://orcid.org/0000-0003-0729-8359

Received: April 1, 2021

Revised: April 29, 2021

Accepted: April 30, 2021

*These authors contributed equally to this work.
Background: The five times sit-to-stand test (5STS) is one of the most commonly used tests to assess the physical performance of lower extremities. This study assessed the correlation between human interpretation (5STS $S_{\text {human }}$ ) and a rule-based algorithm (5STS rule $)$ using instrumented 5STS with two sensors. Methods: We analyzed clinical records of 148 patients who visited the geriatric outpatient clinic of Asan Medical Center between December 2020 and March 2021 and underwent physical performance assessment using the electronic Short Physical Performance Battery (eSPPB) protocol. For STS, time-weight and time-distance curves were constructed using a loadcell and light detection and ranging (LiDAR). We manually assessed the grids of these curves

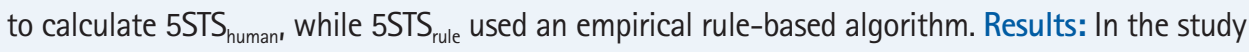
population, the mean $5 S S_{\text {human }}$ and $5 S T S_{\text {rule }}$ times, i.e., $12.2 \pm 0.4$ and $11.4 \pm 0.4$ seconds, respectively, did not differ significantly $(\mathrm{p}=0.232)$. Linear regression analysis showed that $5 \mathrm{STS}$ human and 5STS rule were positively correlated $\left(\beta=0.99, R^{2}=0.99\right)$. The measures also did not differ $(p=0.381)$ in classifying sarcopenia according to the Asian Working Group Society criteria, with $\mathrm{C}$-indices of

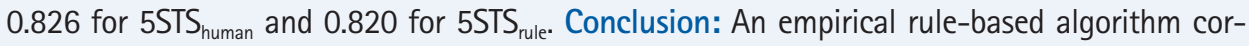
related with human-interpreted 5STS and had comparable classification ability for sarcopenia.

Key Words: Sarcopenia, Physical functional performance, Aging, Biomarkers

\section{INTRODUCTION}

Accumulating evidence suggests the clinical importance of assessing the physical performance of the lower extremities in older adults as a diagnostic marker, ${ }^{1-3)}$ outcome predictor, ${ }^{4-6)}$ and clinical outcome measure. ${ }^{7-9)}$ For example, the assessment of the physical performance of the lower extremities is an essential component in diagnosing sarcopenia, a common geriatric syndrome defined as a state of decreased muscle mass, muscle strength, and/or physical performance. ${ }^{2,10)}$ Longitudinal studies have shown that decreased physical performance of the lower extremities is associated with falls, functional decline, and mortality. ${ }^{11-15)}$ Furthermore, meaningful changes in physical performance following treatment are considered crucial efficacy measures in intervention studies targeting sarcopenia or frailty. ${ }^{16,17)}$

Among the various tools used to assess the physical performance of lower extremities, the five times sit-to-stand test (5STS) is one of the most commonly used tests owing to its advantages in clinical implementation. ${ }^{18)}$ The test can be performed in clinical environments with restricted time and space. ${ }^{19)}$ 5STS is included in the consensus definition for sarcopenia, either as a separate test $(\geq 12$ seconds for impaired physical performance) or as a component of 
the Short Physical Performance Battery (SPPB) score (with scores $\leq 9$ indicating impaired physical performance). ${ }^{2)}$ While most studies have adopted 5STS using a simple chair and stopwatch, researchers have also developed instrumented approaches using sensors of varying modalities to automatically or semi-automatically acquire 5STS measurements to minimize inter-rater variability and collect additional information during test protocols. ${ }^{18-21)}$

We previously developed a multi-sensor kiosk to semi-automatically perform SPPB using light detection and ranging (LiDAR) and loadcells to facilitate physical performance assessments in both clinical and research settings. ${ }^{21)}$ Electronic SPPB (eSPPB) was comparable to SPPB performed manually by a single experienced examiner. ${ }^{21)}$ In addition, continuous parameters derived from eSPPB were used to classify frailty in geriatric outpatients. ${ }^{22}$ ) The eSPPB protocol used two sensors, i.e., a loadcell and LiDAR, as well as a rule-based algorithm, to determine the sit and stand maneuvers. ${ }^{21)}$ Although the correlation between the instrumented 5STS in eSPPB and manual 5STS was assessed semi-quantitatively (score ranging from 0 to 4 ), the validity of the rule-based algorithm has not yet been proven.

Hence, we assessed the correlation between human interpretation $\left(5 S \mathrm{SS}_{\text {human }}\right)$ and the rule-based algorithm $\left(5 \mathrm{STS}_{\text {rule }}\right)$ to determine instrumented 5STS time in geriatric outpatients at a tertiary academic hospital. We also compared the characteristics of these two 5STS measurement methods in classifying sarcopenia.

\section{MATERIALS AND METHODS}

\section{Study Design and Participants}

This study was performed as a retrospective cross-sectional review of clinical records of 165 consecutive outpatients who visited the geriatric outpatient clinic of Asan Medical Center between December 2020 and March 2021 and underwent physical performance examination, including the eSPPB protocol. Community-dwelling and ambulatory patients with or without a walking aid were considered for the test because eSPPB involves gait speed measurement. When performing eSPPB, patients with an estimated life expectancy of less than 1 year owing to advanced malignancy or those with decompensated heart failure or end-stage renal disease, those unable to walk without other persons' assistance, or those with cognitive dysfunction who could not perform eSPPB according to instructions were excluded. We also removed $17 \mathrm{du}-$ plicate records of patients who underwent eSPPB more than once and included 148 records in our final analysis. Among the study population, 5STS and sarcopenia were analyzed in 126 patients who underwent geriatric assessments, including demographic, medical, and sarcopenia parameters.
The protocol for this study was reviewed and approved by the Institutional Review Board of the Asan Medical Center (No. 20210519). Because of the retrospective nature of the study, the requirement for informed consent was waived. We maintained the confidentiality of patients' health information and performed our analysis after anonymizing the dataset. In this study, the researchers complied with the ethical rules for human experimentation stated in the Declaration of Helsinki. ${ }^{23)}$

\section{Measurement of 5STS}

\section{Test protocol and sensor composition}

For 5STS, the patients were instructed to perform five sit-to-stand maneuvers as quickly as possible, with their arms crossed on the shoulders of the opposite side. To acquire 5STS data, we combined two sensors to simultaneously measure the seated weight and position of participants in real time (Fig. 1). First, a loadcell measured the weights of the sitting participants every $10 \mathrm{~ms}$ and produced a time-weight curve. Second, a LiDAR sensor measured the distance from the corner of the chair to the buttock of participants to produce a time-distance curve. These sensors were connected to a computer using the XBee wireless protocol (Digi International Inc., Hopkins, MN, USA) and controlled by a software that performed the standardized eSPPB protocol (Dyphi Inc., Daejeon, Korea). ${ }^{14,21)}$ For the categorization of 5STS, we employed the widely used criterion for SPPB, ranging from 0 to 4 ( 0 for 5STS $>60$ seconds, 1 for $16.7-60$ seconds, 2 for $13.7-16.7$ seconds, 3 for $11.2-13.7$ seconds, and 4 for $\leq 11.2$ seconds).

\section{sSTS $_{\text {human }}$}

Graphs of the time-weight curve and time-distance curve were interpreted by HWJ who was blinded to demographic and clinical parameters of the participants. For interpretation, 200-ms vertical
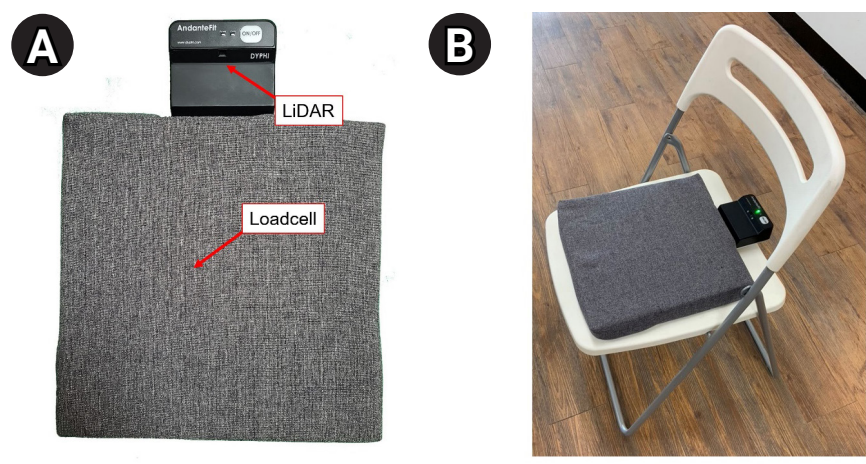

Fig. 1. (A) Layout of the sensors (loadcell and light detection and ranging $[\mathrm{LiDAR}]$ ) used in the study. (B) Sensor attached to a common chair without an armrest. 
grids were introduced in the graphs to determine the time points of (1) the buttock of participants detached from the chair as the starting point and (2) participants completed five chair stands as the finish point (Fig. 2) to best mimic eye observed 5STS. The duration between the start and finish points $\left(5 \mathrm{STS}_{\text {human }}\right)$ was calculated.

\section{SSTS $_{\text {rule }}$}

For $5 \mathrm{STS}_{\text {rule }}$, we used both time-weight and time-distance curves to define four conceptual phases of 5STS: (1) sit, weight $\geq 10 \mathrm{~kg}$; (2) sit-to-stand, weight $<10 \mathrm{~kg}$ and distance $<30 \mathrm{~cm}$; (3) stand, weight $<1.5 \mathrm{~kg}$ and distance $>30 \mathrm{~cm}$; and (4) stand-to-sit, weight $\geq 1.5 \mathrm{~kg}$. The cut-off values for weight and distance were established empirically. Based on these rules, the starting point of the test was defined as an initial seated weight decrement of $<10 \mathrm{~kg}$, while the ending point of the test was indicated by the fifth increment of distance exceeding $30 \mathrm{~cm}$ (Fig. 2).

\section{Sarcopenia Assessments}

Muscle mass was assessed using bioelectrical impedance analysis (InBody S10; InBody, Seoul, Korea). Appendicular skeletal muscle (ASM) was estimated by summing the muscle masses of the four extremities. ASM was divided by height ${ }^{2}\left(\mathrm{~m}^{2}\right)$ to calculate the skeletal muscle mass index (SMI) to adjust for anthropometric differences among patients. Grip strength of the dominant hand was measured using a JAMAR hydraulic handgrip dynamometer (Patterson Medical, Warrenville, IL, USA) with the elbow flexed at $90^{\circ}$ and the participant in a seated position. For walking speed, we measured the 4-m usual gait speed, with a separate 1-m acceleration distance that was excluded from the speed calculation. ${ }^{24)}$

Sarcopenia was determined according to the 2019 Asian Working Group for Sarcopenia (AWGS) guidelines, in which individuals with low muscle mass (SMI $<7.0 \mathrm{~kg} / \mathrm{m}^{2}$ for men and $<5.7 \mathrm{~kg}$ ) $\mathrm{m}^{2}$ for women), low muscle strength (grip strength $<28 \mathrm{~kg}$ for men and $<18 \mathrm{~kg}$ for women), or low physical performance (gait speed $<1.0 \mathrm{~m} / \mathrm{s}$ ) were considered as having sarcopenia.

\section{Statistical Analysis}

Descriptive statistics were analyzed for demographic and geriatric parameters of the study population. T-tests and chi-square tests were used to compare continuous and categorical variables, respectively. We assessed the intraclass correlation coefficient (ICC) between 5 STS $_{\text {human }}$ and 5STS rule and used a scatterplot with linear regression analysis to assess the correlation between $5 \mathrm{STS}_{\text {human }}$ and

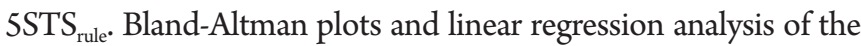
means and differences were used to evaluate the potential biases between these two measures, with the absolute difference as a dependent variable and the average as an independent variable. ${ }^{25)}$ The kappa statistic was calculated to assess the agreement between categorized $5 \mathrm{STS}_{\text {human }}$ and $5 \mathrm{STS}$ rule scores. The performance of $5 S T S_{\text {human }}$ and $5 S T S_{\text {rule }}$ in classifying sarcopenia was assessed by receiver operating characteristic (ROC) analysis and comparisons of $\mathrm{C}$-indices. Two-sided $\mathrm{p}$-values $<0.05$ were considered statistically significant. Analyses were performed using Stata 16.0 (StataCorp, College Station, TX, USA).
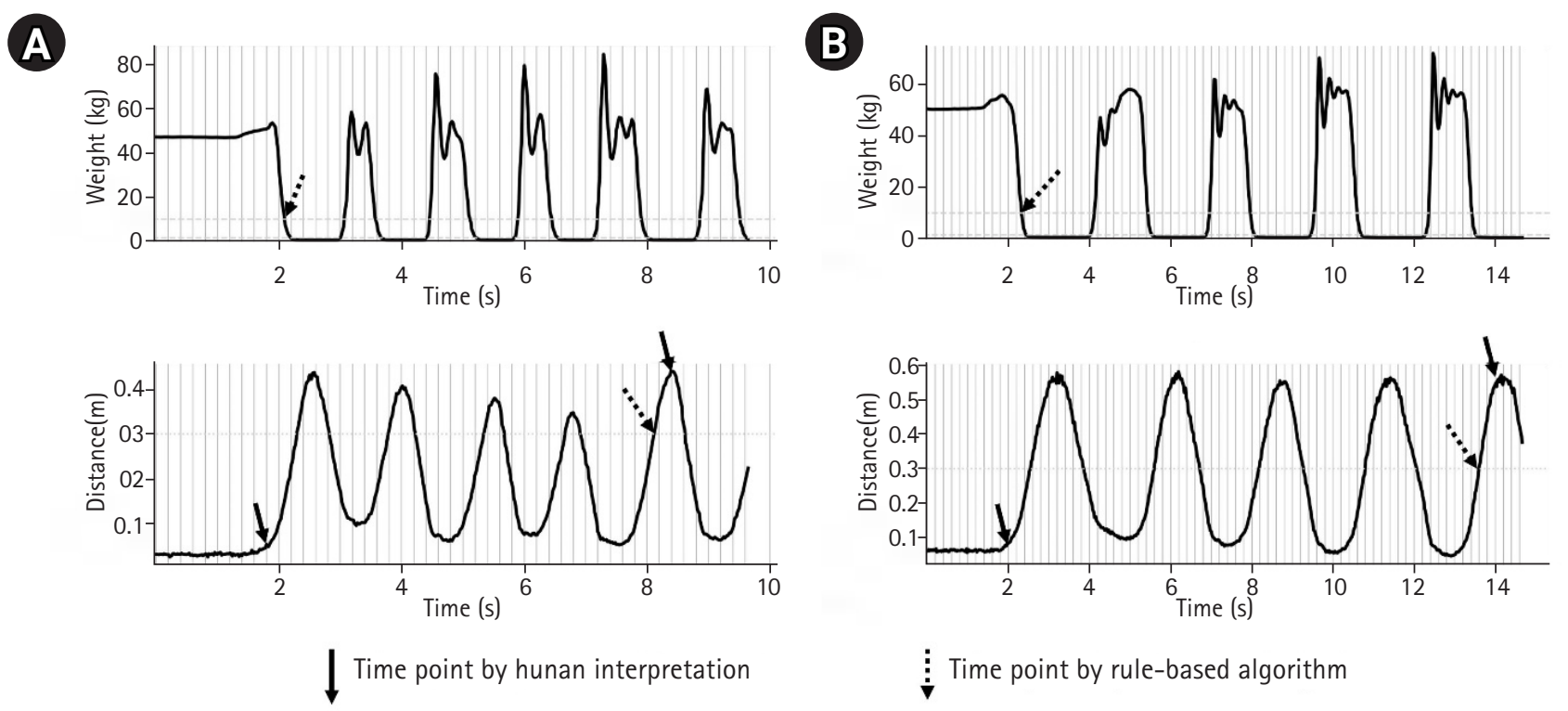

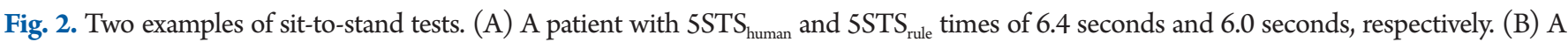

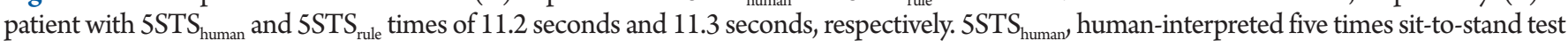
time; $5 \mathrm{STS}_{\text {rule }}$, rule-based five times sit-to-stand test. 


\section{RESULTS}

\section{Basic Characteristics}

In the study population, the mean age was $76.3 \pm 7.6$ years, and 88 outpatients $(59.5 \%)$ were female (Table 1$)$. The 5 STS $_{\text {human }}$ time was significantly shorter $(\mathrm{p}=0.035)$ in men ( $11.0 \pm 3.3$ seconds $)$ than in women (12.9 \pm 6.4 seconds). Similarly, the $5 S$ TS $_{\text {rule }}$ time was significantly shorter $(\mathrm{p}=0.026)$ in men $(10.3 \pm 3.2$ seconds $)$ than in women ( $12.2 \pm 6.0$ seconds). Age was positively correlated with both 5 STS $_{\text {human }}$ (standardized beta $[\beta]=0.30, \mathrm{p}<0.001$ ) and $5 \operatorname{STS}_{\text {rule }}(\beta=0.29, \mathrm{p}<0.001)$. The worst quintile values of 5 STS $_{\mathrm{hu}-}$ man and 5 STS $_{\text {rule }}$ were 14.8 and 13.7 seconds, respectively.

\section{Agreements between 5STS $_{\text {human }}$ and 5STS ${ }_{\text {rule }}$}

The absolute difference between STS by 5 STS $_{\text {human }}(12.2 \pm 0.4 \mathrm{sec}-$ onds) and $5 \operatorname{STS}_{\text {rule }}(11.4 \pm 0.4$ seconds) was $0.74 \pm 0.62$ seconds, and it did not differ significantly $(\mathrm{p}=0.232)$. The correlations between these two parameters are shown in Fig. 1. Linear regression analysis showed that $5 \mathrm{STS}_{\text {human }}$ and $5 \mathrm{STS}$ rule were positively correlated $\left(\beta=0.99, \mathrm{R}^{2}=0.99, \mathrm{p}<0.001,5 \mathrm{STS}_{\text {human }}=1.05 \times 5 \mathrm{STS}\right.$ rule +0.18$)$. The ICC of the average measures between $5 S_{\text {TS }}$ and 5 STS $_{\text {rule }}$ was $0.98(\mathrm{p}<0.001)$. A proportional bias between 5STS $_{\text {human }}$ and $5 \mathrm{STS}$ rule was observed using linear regression (Fig. 3, $\left.\beta=0.45, R^{2}=0.19, p<0.001\right)$.

Since 5STS is commonly performed as a component of SPPB, we compared the categorical scores between 5 STS $_{\text {human }}$ and 5STSrule (Table 2). The kappa statistic between the two measures was 0.88 , suggesting a substantial agreement.

\section{Performance of Classifying Sarcopenia}

Among 126 patients who underwent assessments for sarcopenia,

Table 1. Basic characteristics of the study population

\begin{tabular}{lccr}
\hline & Male $(\mathrm{n}=60)$ & Female $(\mathrm{n}=88)$ & p-value \\
\hline Age $(\mathrm{y})$ & $75.1 \pm 9.5$ & $77.0 \pm 5.9$ & 0.132 \\
$\operatorname{SSTS}_{\text {human }}(\mathrm{s})$ & $11.0 \pm 3.3$ & $12.9 \pm 6.4$ & 0.035 \\
$\operatorname{SSTS}_{\text {rule }}(\mathrm{s})$ & $10.3 \pm 3.2$ & $12.2 \pm 6.0$ & 0.026 \\
$\mathrm{BMI}^{2}\left(\mathrm{~kg} / \mathrm{m}^{2}\right)$ & $23.7 \pm 3.2$ & $25.4 \pm 6.5$ & 0.088 \\
$\mathrm{ASM}^{2 \mathrm{ht}^{2}}\left(\mathrm{~kg} / \mathrm{m}^{2}\right)$ & $7.1 \pm 0.85$ & $5.8 \pm 0.7$ & $<0.001$ \\
$\mathrm{Grip} \mathrm{strength}^{\mathrm{a})}(\mathrm{kg})$ & $34.0 \pm 8.5$ & $20.3 \pm 5.4$ & $<0.001$ \\
Gait speed $^{\mathrm{a})}(\mathrm{m} / \mathrm{s})$ & $0.96 \pm 0.28$ & $0.88 \pm 0.28$ & 0.070 \\
Hypertensiona $^{2}(\%)$ & $25(53.2)$ & $53(67.1)$ & 0.120 \\
Diabetes $^{\text {a) }}(\%)$ & $15(31.9)$ & $33(41.8)$ & 0.271 \\
Fall history in 1 year $^{\mathrm{a})}(\%)$ & $4(8.5)$ & $15(19.0)$ & 0.122
\end{tabular}

Values are presented as mean \pm standard deviation or number (\%).

$5 \mathrm{STS}_{\text {human }}$, human-interpreted five times sit-to-stand test time; $5 \mathrm{STS}$ rule rulebased five times sit-to-stand test time; BMI, body mass index; ASM $/ \mathrm{ht}^{2}$, appendicular skeletal muscle mass adjusted by height.

a) Available from 47 male and 79 female patients.
34 (27.0\%) met the AWGS criteria for sarcopenia. Specifically, 58 (46.0\%) had low muscle mass, 42 (33.3\%) had low gait speed, and 33 (26.2\%) had low grip strength. In ROC analysis (Fig. 4), the $\mathrm{C}$-indices for classifying sarcopenia according to the AWGS criteria from $5 \mathrm{STS}_{\text {human }}$ and $5 \mathrm{STS}$ rule were 0.826 (95\% confidence interval [CI], 0.749-0.903) and 0.820 (95\% CI, 0.743-0.897), respectively, showing no statistically significant difference $(\mathrm{p}=0.381)$. The cut-off times with maximal sensitivity+specificity for sarcopenia were $\geq 13.8$ seconds for 5 STS $_{\text {human }}$ and $\geq 12.8$ seconds for 5STS rule .

\section{DISCUSSION}

The results of this study demonstrated the correlation between

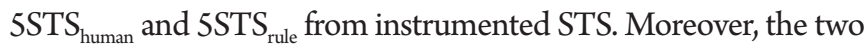
approaches showed similar abilities in detecting sarcopenia. While the mean difference between 5 STS ${ }_{\text {human }}$ and 5 STS ${ }_{\text {rule }}$ was not signif-

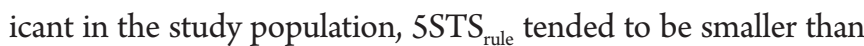
$5 S$ SS $_{\text {human }}$ with intrinsic characteristics of empirically determined rule-based criteria defining the phase shift between the sit-to-stand and standing states, in contrast to human interpretation, which tried to capture the maximal peak of the distance between the LiDAR sensor and patients. To our knowledge, this study is the first attempt to adopt a type of sensor fusion method using both $\mathrm{Li}$ DAR and loadcells to assess 5STS.

Previous studies have attempted to capture the dynamics of 5STS using different sensors such as accelerometers, motion capture devices, force plates, depth cameras, and RGB cameras. ${ }^{18-20,26-29)}$ Shukla et al. ${ }^{19)}$ showed that a force plate or loadcell-embedded seat could more accurately capture 5STS than depth or RGB cameras, with a smaller mean error size compared to a human expert examiner. In the study, ${ }^{19)}$ the results of the phase-determination rule-based algorithm interpreting the time-weight curve of a loadcell-embedded chair were generally similar, although researchers adopted a percent cut-off value to define phases rather

Table 2. Agreement between categorized $5 \mathrm{STS}_{\text {human }}$ and $5 \mathrm{STS}_{\text {rule }}$ scores $(0$ for $5 S T S>60$ s, 1 for $16.7-60$ s, 2 for $13.7-16.7$ s, 3 for $11.2-13.7 \mathrm{~s}$, and 4 for $\leq 11.2 \mathrm{~s}$ )

\begin{tabular}{lccccc}
\hline 5STS $_{\text {rule }}$ & \multicolumn{5}{c}{ 5STS $_{\text {human }}$} \\
\cline { 2 - 6 } & 1 & 2 & 3 & 4 & Total \\
\hline 1 & 20 & 0 & 0 & 0 & 20 \\
2 & 2 & 8 & 0 & 0 & 10 \\
3 & 0 & 9 & 17 & 1 & 27 \\
4 & 0 & 0 & 6 & 85 & 91 \\
Total & 22 & 17 & 23 & 86 & 148 \\
\hline
\end{tabular}

$5 S \mathrm{SS}_{\text {human }}$, human-interpreted five times sit-to-stand test time; $5 \mathrm{STS}$ rule, rulebased five times sit-to-stand test. 

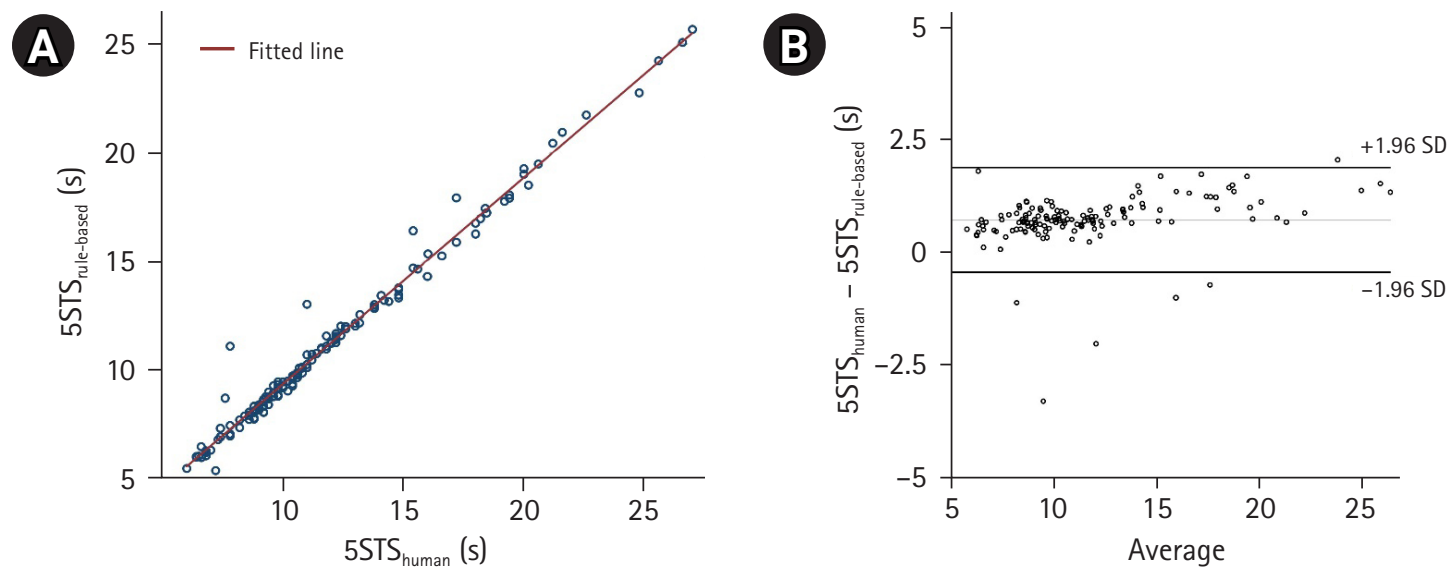

Fig. 3. (A) Scatterplot showing 5 STS $_{\text {human }}$ and 5 STS $_{\text {rule }}$ times and their correlations according to a fitted line. (B) Bland-Altman plot of 5STS human $_{\text {A }}$

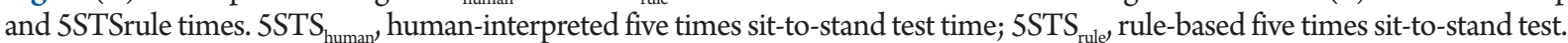

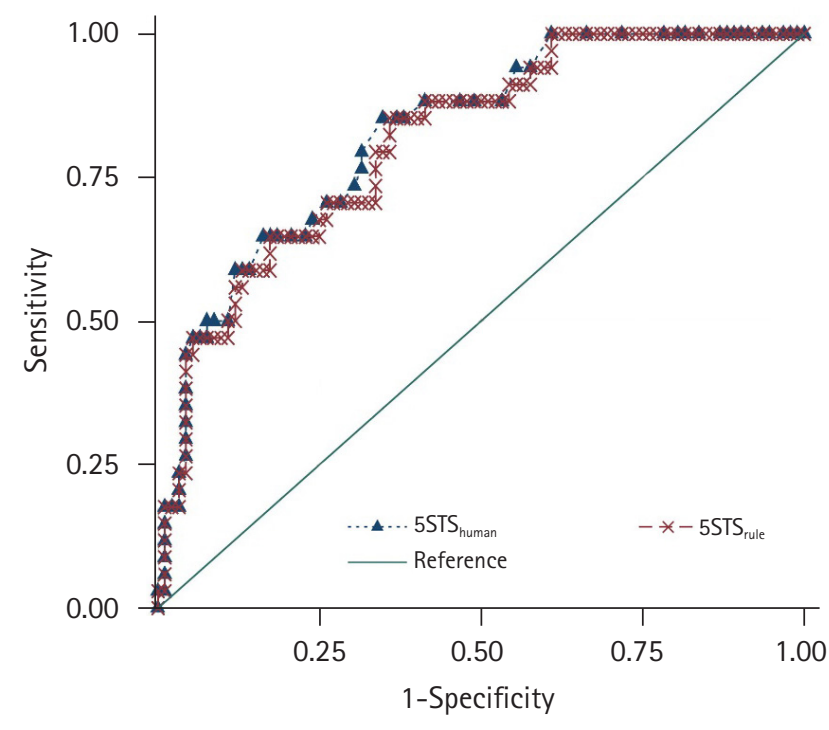

Fig. 4. Receiver operating characteristic curves of 5 STS $_{\text {human }}$ and 5STS $_{\text {rule }}$ in classifying sarcopenia according to the Asian Working Group Society guideline. $5 S$ SS $_{\text {human }}$, human-interpreted five times sitto-stand test time; $5 \mathrm{STS}_{\text {rule }}$, rule-based five times sit-to-stand test.

than the absolute weight value as used in the present study. Although a single loadcell sensor could reliably measure STS, as demonstrated by Shukla et al. ${ }^{19)}$, we noted that rule-based algorithms based on loadcell data were sometimes less reliable in capturing STS phases, especially in frail and underweight female patients in real-world settings. To address this issue, we added a LiDAR set at $45^{\circ}$ to further acquire time-distance data by tracking the distance between the sensor and buttock of the participants. In contrast to non-wearable sensor approaches, studies have also adopted wearable sensors. ${ }^{26,27)}$ However, these studies generally focused on exploring new features to assess fall risk rather than measuring 5STS per se.
We observed that the rule-based interpretation provided more rapid 5STS, with an absolute difference of 0.7 seconds, compared to that for $5 \mathrm{STS}_{\text {human }}$. A previous report showed a minimally detectable 5STS difference of 2.5 seconds ${ }^{30)}$ thus, the difference between $5 \mathrm{STS}_{\text {human }}$ and $5 \mathrm{STS}$ rule is less likely to affect the interpretation of the physical performance of patients in clinical assessments. Our observation of agreement between the categorized scores of $5 \mathrm{STS}_{\text {human }}$ and $5 \mathrm{STS}$ rule further supports the comparability of these two approaches. However, to alleviate possible concerns regarding differences owing to the slightly biased interpretation of 5STS rule regression equation between $5 \mathrm{STS}_{\text {human }}$ and $5 \mathrm{STS} \mathrm{S}_{\text {rule }}$ can be performed to calculate the estimated $5 \mathrm{STS}_{\text {human }}$ using the automatically measured $5 \mathrm{STS}$ rule

Although we used an empirically established rule-based algorithm in the current study, there is still room for improvement in optimally mimicking $5 \mathrm{STS}_{\text {human }}$. Given sufficient data from patients with a wide spectrum of physical performance and expert annotation on time-weight and time-distance curves, machine learning can be used to develop better algorithms. With the development of improved algorithms, instrumented 5STS can be more confidently adopted both as a screening tool and as an outcome measure with minimal inter-rater variability for intervention schemes targeting sarcopenia.

The strengths of this study are the implementation of sensors and algorithms for real-world geriatric outpatients and the clinical assessments of sarcopenia. While eSPPB using these sensors has been in clinical use since 2019, raw weight-time and distance-time data other than the eSPPB score were not stored in the hardware until a software update in December 2020 while the updated software allowed the current post-hoc analysis for 5STS using stored raw eSPPB data. Similar to a previous study using continuous parameters of eSPPB for classifying frailty, ${ }^{22)}$ explorations of human 
kinetic features with newer algorithms using already installed sensors may identify additional physical biomarkers related to sarcopenia and frailty.

The present study had some limitations. Most importantly, STS was performed only using the instrument while manually measured 5STS using the human eye and stopwatch was not available since the study was a retrospective analysis using stored eSPPB data. However, as previously demonstrated, ${ }^{19)}$ 5STS by human observation is unlikely to affect the results of the current study. In addition, as a single-center study in a tertiary academic hospital in Korea, the generalizability of the results is limited. The statistical significance of the absolute difference between 5 STS $S_{\text {human }}$ and 5STS ${ }_{\text {rule }}$ cannot be ruled out if the study was performed with sufficiently large sample size. To address these issues, future studies with larger populations of diverse ethnicities are warranted.

In conclusion, $5 S \mathrm{SS}_{\text {human }}$ and $5 \mathrm{STS}$ rule times using a LiDAR and loadcell were correlated in older geriatric outpatients. Both measures agreed with the categorical scores of the SPPB and had comparable classification ability for sarcopenia. Our findings support clinical evidence for adopting instrumented 5STS in clinical practice and research for older adults.

\section{ACKNOWLEDGMENTS}

\section{CONFLICT OF INTEREST}

Hee-Won Jung, Seongjun Yoon, and Hyunchul Roh cofounded Dyphi Inc., a startup company on sensor technology. The other researchers claim no conflicts of interest.

\section{FUNDING}

This research was supported by a grant from the Korea Health Technology R\&D Project through the Korea Health Industry Development Institute (KHIDI) funded by the Ministry of Health \& Welfare, Republic of Korea (No. HI18C2383). The sponsor played no role in any part of this study, including the design, methods, recruitment, data collection, analysis, and manuscript preparation.

\section{AUTHOR CONTRIBUTIONS}

Conceptualization, HWJ, SY, HR; Data curation, HWJ, SY, IYJ, HR; Funding acquisition, IYJ; Investigation, HWJ, SY, HR; Methodology, HWJ, SY, IYJ, HR; Writing-original draft, HWJ; Writing-review \& editing, all.

\section{REFERENCES}

1. Cruz-Jentoft AJ, Bahat G, Bauer J, Boirie Y, Bruyere O, Cederholm T, et al. Sarcopenia: revised European consensus on defini- tion and diagnosis. Age Ageing 2019;48:16-31.

2. Chen LK, Woo J, Assantachai P, Auyeung TW, Chou MY, Iijima K, et al. Asian Working Group for Sarcopenia: 2019 Consensus update on sarcopenia diagnosis and treatment. J Am Med Dir Assoc 2020;21:300-307.e2.

3. Jung HW, Kim S, Jang IY, Shin DW, Lee JE, Won CW. Screening value of timed up and go test for frailty and low physical performance in Korean older population: the Korean Frailty and Aging Cohort Study (KFACS). Ann Geriatr Med Res 2020;24:259-66.

4. Volpato S, Cavalieri M, Sioulis F, Guerra G, Maraldi C, Zuliani G, et al. Predictive value of the Short Physical Performance Battery following hospitalization in older patients. J Gerontol A Biol Sci Med Sci 2011;66:89-96.

5. Vasunilashorn S, Coppin AK, Patel KV, Lauretani F, Ferrucci L, Bandinelli S, et al. Use of the Short Physical Performance Battery Score to predict loss of ability to walk 400 meters: analysis from the InCHIANTI study. J Gerontol A Biol Sci Med Sci 2009;64: 223-9.

6. Lee JE, Chun H, Kim YS, Jung HW, Jang IY, Cha HM, et al. Association between timed up and go test and subsequent functional dependency.J Korean Med Sci 2020;35:e25.

7. Rooks D, Swan T, Goswami B, Filosa LA, Bunte O, Panchaud N, et al. Bimagrumab vs optimized standard of care for treatment of sarcopenia in community-dwelling older adults: a randomized clinical trial. JAMA Netw Open 2020;3:e2020836.

8. Park CM, Oh G, Lee H, Jung HW, Lee E, Jang IY, et al. Multicomponent intervention and long-term disability in older adults: a nonrandomized prospective study. J Am Geriatr Soc 2021; 69:669-677.

9. Jang IY, Jung HW, Park H, Lee CK, Yu SS, Lee YS, et al. A multicomponent frailty intervention for socioeconomically vulnerable older adults: a designed-delay study. Clin Interv Aging 2018;13:1799-814.

10. Jang HC. Diabetes and muscle dysfunction in older adults. Ann Geriatr Med Res 2019;23:160-4.

11. Pavasini R, Guralnik J, Brown JC, di Bari M, Cesari M, Landi F, et al. Short Physical Performance Battery and all-cause mortality: systematic review and meta-analysis. BMC Med 2016;14:215.

12. Guralnik JM, Ferrucci L, Pieper CF, Leveille SG, Markides KS, Ostir GV, et al. Lower extremity function and subsequent disability: consistency across studies, predictive models, and value of gait speed alone compared with the short physical performance battery. J Gerontol A Biol Sci Med Sci 2000;55:M221-31.

13. Guralnik JM, Ferrucci L, Simonsick EM, Salive ME, Wallace RB. Lower-extremity function in persons over the age of 70 years as a predictor of subsequent disability. N Engl J Med 1995;332:55661. 
14. GuralnikJM, Simonsick EM, Ferrucci L, Glynn RJ, Berkman LF, Blazer DG, et al. A short physical performance battery assessing lower extremity function: association with self-reported disability and prediction of mortality and nursing home admission. J Gerontol 1994;49:M85-94.

15. Jung HW, Jang IY, Lee CK, Yu SS, Hwang JK, Jeon C, et al. Usual gait speed is associated with frailty status, institutionalization, and mortality in community-dwelling rural older adults: a longitudinal analysis of the Aging Study of Pyeongchang Rural Area. Clin Interv Aging 2018;13:1079-89.

16. Guralnik J, Bandeen-Roche K, Bhasin SA, Eremenco S, Landi F, Muscedere J, et al. Clinically meaningful change for physical performance: perspectives of the ICFSR Task Force. J Frailty Aging 2020;9:9-13.

17. High KP, Zieman S, Gurwitz J, Hill C, Lai J, Robinson T, et al. Use of functional assessment to define therapeutic goals and treatment. J Am Geriatr Soc 2019;67:1782-90.

18. Shukla B, Bassement J, Vijay V, Yadav S, Hewson D. Instrumented analysis of the sit-to-stand movement for geriatric screening: a systematic review. Bioengineering (Basel) 2020;7:139.

19. Shukla BK, Jain H, Vijay V, Yadav SK, Mathur A, Hewson DJ. A comparison of four approaches to evaluate the sit-to-stand movement. IEEE Trans Neural Syst Rehabil Eng 2020;28:131724.

20. Hellec J, Chorin F, Castagnetti A, Colson SS. Sit-to-stand movement evaluated using an inertial measurement unit embedded in smart glasses: a validation study. Sensors (Basel) 2020;20:5019.

21. Jung HW, Roh H, Cho Y, Jeong J, Shin YS, Lim JY, et al. Validation of a multi-sensor-based kiosk for short physical performance battery.J Am Geriatr Soc 2019;67:2605-9.
22. Jung HW, Jin T, Baek JY, Yoon S, Lee E, Guralnik JM, et al. Functional age predicted by electronic short physical performance battery can detect frailty status in older adults. Clin Interv Aging 2020;15:2175-82.

23. World Medical Association. World Medical Association Declaration of Helsinki: ethical principles for medical research involving human subjects. Bull World Health Organ 2001;79:373-4.

24. Jung HW, Roh HC, Kim SW, Kim S, Kim M, Won CW. Cross-comparisons of gait speeds by automatic sensors and a stopwatch to provide converting formula between measuring modalities. Ann Geriatr Med Res 2019;23:71-6.

25. Ho KM. Using linear regression to assess dose-dependent bias on a Bland-Altman plot.J Emerg Crit Care Med 2018;2:68.

26. Doheny EP, Fan CW, Foran T, Greene BR, Cunningham C, Kenny RA. An instrumented sit-to-stand test used to examine differences between older fallers and non-fallers. Annu Int Conf IEEE Eng Med Biol Soc 2011;2011:3063-6.

27. Doheny EP, Walsh C, Foran T, Greene BR, Fan CW, Cunningham $\mathrm{C}$, et al. Falls classification using tri-axial accelerometers during the five-times-sit-to-stand test. Gait Posture 2013;38: 1021-5.

28. Chorin F, Cornu C, Beaune B, Frere J, Rahmani A. Sit to stand in elderly fallers vs non-fallers: new insights from force platform and electromyography data. Aging Clin Exp Res 2016;28:871-9.

29. Hellmers S, Fudickar S, Lau S, Elgert L, Diekmann R, Bauer JM, et al. Measurement of the chair rise performance of older people based on force plates and IMUs. Sensors (Basel) 2019;19:1370.

30. Goldberg A, Chavis M, Watkins J, Wilson T. The five-times-sitto-stand test: validity, reliability and detectable change in older females. Aging Clin Exp Res 2012;24:339-44. 